ARTICLE

Received 29 Feb 2016 | Accepted 18 Aug 2016 | Published 22 Sep 2016

DOI: $10.1038 /$ ncomms12952

OPEN

\title{
Unusual lattice vibration characteristics in whiskers of the pseudo-one-dimensional titanium trisulfide $\mathrm{TiS}_{3}$
}

\author{
Kedi Wu' ${ }^{1}$, Engin Torun ${ }^{2}$, Hasan Sahin ${ }^{2,3}$, Bin Chen ${ }^{1}$, Xi Fan ${ }^{1}$, Anupum Pant ${ }^{1}$, David Parsons Wright ${ }^{4}$,
} Toshihiro Aoki ${ }^{4}$, Francois M. Peeters ${ }^{2}$, Emmanuel Soignard ${ }^{4} \&$ Sefaattin Tongay ${ }^{1}$

Transition metal trichalcogenides form a class of layered materials with strong in-plane anisotropy. For example, titanium trisulfide $\left(\mathrm{TiS}_{3}\right)$ whiskers are made out of weakly interacting $\mathrm{TiS}_{3}$ layers, where each layer is made of weakly interacting quasi-one-dimensional chains extending along the $b$ axis. Here we establish the unusual vibrational properties of $\mathrm{TiS}_{3}$ both experimentally and theoretically. Unlike other two-dimensional systems, the Raman active peaks of $\mathrm{TiS}_{3}$ have only out-of-plane vibrational modes, and interestingly some of these vibrations involve unique rigid-chain vibrations and $\mathrm{S}-\mathrm{S}$ molecular oscillations. High-pressure Raman studies further reveal that the $A_{g}^{S-S}$ S-S molecular mode has an unconventional negative pressure dependence, whereas other peaks stiffen as anticipated. Various vibrational modes are doubly degenerate at ambient pressure, but the degeneracy is lifted at high pressures. These results establish the unusual vibrational properties of $\mathrm{TiS}_{3}$ with strong in-plane anisotropy, and may have relevance to understanding of vibrational properties in other anisotropic two-dimensional material systems.

\footnotetext{
${ }^{1}$ School for Engineering of Matter, Transport and Energy, Arizona State University, Tempe, Arizona 85287, USA. ${ }^{2}$ Department of Physics, University of Antwerp, Groenenborgerlaan 171, Antwerpen B-2020, Belgium. ${ }^{3}$ Department of Photonics, Izmir Institute of Technology, Izmir 35430, Turkey. ${ }^{4}$ LeRoy Eyring Center for Solid State Science, Arizona State University, Tempe, Arizona 85287, USA. Correspondence and requests for materials should be addressed to S.T. (email: Sefaattin.tongay@asu.edu).
} 
$\mathrm{T}$ wo-dimensional (2D)-layered materials have emerged as a new class of materials with unusual optical, electrical, mechanical and thermal properties. Owing to their unique properties, they are of potential interest for applications for energy conversion, flexible electronics and information technologies $^{1-5}$. Lamellar materials consist of two-dimensional (2D) layers weakly coupled through van der Waals (vdW) interaction; thus, they possess large structural anisotropy in the $c$ axis (out-of-plane direction), whereas the individual 2D layer has high in-plane isotropy. However, there exist a small number of $2 \mathrm{D}$ materials in which one-dimensional (1D) chain-like dimerization takes place to induce strong in-plane anisotropy, such as few-layer black phosphorus ${ }^{6,7}$ and $\mathrm{ReS}_{2}$ $($ refs 8,9$)$. As a result of this anisotropy, their properties vastly differ in different crystalline ( $a$ versus $b$ axis) directions, and offer unique functionalities compared with isotropic $2 \mathrm{D}$ materials ${ }^{10}$.

Layered group-IV transition metal trichalcogenides (TMTCs) with the chemical formula of $\mathrm{MX}_{3}$, for example, $\mathrm{M}=\mathrm{Zr}$, Ti and $\mathrm{X}=\mathrm{S}, \mathrm{Se}$, are known to be anisotropic $2 \mathrm{D}$ systems ${ }^{11}$. Indeed, individual $\mathrm{MX}_{3}$ layers are made out of moderately interacting 1D-like chain structures, which result in large structural in-plane anisotropy ${ }^{12-14}$. Recently, titanium trisulfide $\left(\mathrm{TiS}_{3}\right)$ attracted interest owing to theoretically calculated $1.0 \mathrm{eV}$ direct gap values $^{15-18}$, and field effect transistors have been demonstrated with relatively large electronic mobility ${ }^{19,20}$. Owing to its strong in-plane anisotropy, $\mathrm{TiS}_{3}$ nanosheets measure polarized carrier mobility of $80 \mathrm{~cm}^{2} \mathrm{~V}^{-1} \mathrm{~s}^{-1}$ along the $b$ axis, whereas $40 \mathrm{~cm}^{2} \mathrm{~V}^{-1} \mathrm{~s}^{-1}$ along the $a$ axis $^{21}$. Despite these recent studies, its fundamental vibrational properties, that is, the origin and behaviour of experimentally measured Raman peaks and theoretically calculated vibrational dispersion, remain largely unknown. For example, all prominent $\mathrm{TiS}_{3}$ Raman modes are identified as $\mathrm{A}_{\mathrm{g}}$-like 'out-of-plane' vibrations without further insight into their origin and behaviour ${ }^{22,23}$. Lack of fundamental understanding of their vibrational properties limits our ability to explore their full potential in variety of applications and determination of their properties, such as thermal transport, interface interaction/physics, strain/mechanical behaviour and defect/impurity quantification.

In this work, we report on the unusual vibrational properties of $\mathrm{TiS}_{3}$ whiskers through pressure-dependent diamond anvil cell (DAC)-Raman spectroscopy measurements and density functional theory (DFT) calculations. In brief, results show that four prominent $\mathrm{TiS}_{3}$ Raman peaks located at $\sim 176,298,370$ and $556 \mathrm{~cm}^{-1}$ are associated with rigid chain vibrations (I-A $A_{\mathrm{g}}^{\text {rigid }}$ ), internal out-of-plane vibrations constituting each monolayer (II- $\mathrm{A}_{\mathrm{g}}^{\text {internal }}$ and III- $\mathrm{A}_{\mathrm{g}}^{\text {internal }}$ ) and sulfur-sulfur diatomic motions (IV- $\mathrm{A}_{\mathrm{g}}^{\mathrm{s}-\mathrm{s}}$ ), respectively, which is in stark contrast to other isotropic $2 \mathrm{D}$ materials. Interestingly, DAC high-pressure Raman studies (up to $\sim 26 \mathrm{GPa}$ ) reveal that all Raman modes stiffen with pressure $(\mathrm{d} \omega / \mathrm{d} P>0)$ as commonly anticipated, except that the IV-A $A_{\mathrm{g}}^{S-S}$ mode softens $(\mathrm{d} \omega / \mathrm{d} P<0)$ because of weakening S-S interaction with pressure. Moreover, the II- $\mathrm{A}_{\mathrm{g}}^{\text {internal }}$ and IV- $\mathrm{A}_{\mathrm{g}}^{\mathrm{s}-\mathrm{s}}$ modes split into two individual peaks at pressures well below that required for inducing phase transition. Theoretical studies suggest that these two modes are degenerate at ambient pressure, but hydrostatic pressure lifts the degeneracy due to different $\mathrm{d} \omega / \mathrm{d} P$ values of each peak. This work, to our knowledge, marks the very first DAC measurements on $\mathrm{TiS}_{3}$ and introduces theoretically calculated phonon dispersion of $\mathrm{TiS}_{3}$ pseudo-1D systems. The findings also advance broader understanding of vibrational characteristics of TMTC materials with a potential impact on exploring and interpreting their thermal behaviour, mechanical properties, thermoelectric response and structural properties.

\section{Results}

$\mathrm{TiS}_{3}$ whisker growth and characterization. $\mathrm{TiS}_{3}$ whiskers were grown directly from the interaction between titanium and sulfur in a sealed evacuated quartz ampule at $500^{\circ} \mathrm{C}$ for 4 days via a chemical vapour transport process ${ }^{13,24}$. Typical growth yields $\mathrm{TiS}_{3}$ on the interior wall of the quartz ampule in a whisker form because of its highly anisotropic structure, and the whiskers measures from $\sim 200$ to $500 \mu \mathrm{m}$ in length (Fig. 1a) and only a few microns in width. In this work, most of the measurements were performed on flakes that measured $\sim 5 \mu \mathrm{m}$ in width, $>50 \mu \mathrm{m}$ in length and $\sim 10-100 \mathrm{~nm}$ in thickness. $\mathrm{TiS}_{3}$ crystallizes in the monoclinic $\mathrm{P} 2{ }_{1} / \mathrm{m}$ space group with each unit cell containing two titanium atoms and six sulfur atoms by two distorted prisms, in which the titanium atom is at the top vertex of the prism, and the three sulfur atoms are at the three bottom vertices. The two prisms in the unit cell are also connected by binding the titanium atoms with their closest sulfur atoms in the neighbour prism (Fig. 1b). The lattice constants are calculated as $a=4.98 \AA$, $b=3.39 \AA$ and $c=8.89 \AA$. Here we note two types of sulfur atoms in a single prism: (1) the bridge $S$ atom that is bonded to the two $\mathrm{Ti}$ atoms acting as a bridge and (2) the remaining two $\mathrm{S}$ atoms that are only bonded to only one $\mathrm{Ti}$ atom. To clarify, we differentiate these two types of sulfur atoms and label them as bridge sulfur and sulfur-sulfur pair when discussing the vibrational properties of $\mathrm{TiS}_{3}$. The repeating parallel chains of these triangular prisms are weakly coupled to each other via vdW interaction, giving rise to its signature structural in-plane anisotropy. Figure 1c,d shows the optical and atomic force microscopy (AFM) image of exemplary $\mathrm{TiS}_{3}$ whisker studied in this work. It is clear from these images that $\mathrm{TiS}_{3}$ whiskers have large geometric anisotropy, and the extending chains along the $b$ axis are responsible for the anisotropic nature of the material. Figure le shows the high-resolution transmission electron microscopy (HR-TEM) image of the $\mathrm{TiS}_{3}$. The plane spacing measures $\sim 0.49$ and $\sim 0.33 \mathrm{~nm}$, which agrees well with the distance along the $\langle 100\rangle$ and $<010\rangle$ lattice directions when the sample is aligned with the (011) crystal plane (JCPDS-ICDD 15-0783). The X-ray powder diffraction pattern (Fig. 1f) further confirms the high crystallinity of the synthesized $\mathrm{TiS}_{3}$.

Vibrational properties and phonon dispersion. Raman spectroscopy measurements reveal four prominent peaks located at $176,298,370$ and $556 \mathrm{~cm}^{-1}$ (Fig. 2a). Since a primitive unit cell of $\mathrm{TiS}_{3}$ contains two titanium and six sulfur atoms, the phonon dispersion yields three acoustic and twenty-one optical modes in the first Brillouin zone as shown in Fig. 2b. These peaks match reasonably well with the calculated phonon dispersion spectrum, in particular to those Raman active branches of $\mathrm{TiS}_{3}$ whiskers highlighted by blue diamonds. To understand the vibrational nature of these Raman active modes, finite displacement method is performed and the characteristic atomic motion of each branch is shown in Fig. 2c.

Displacement method results show that lowest frequency peak $\left(176 \mathrm{~cm}^{-1}\right)$ originates from out-of-phase rigid vibration of each $1 \mathrm{D}$-like $\mathrm{TiS}_{3}$ chain extending along the $b$ axis (Fig. 2c labelled I). Since these chains move in the out-of-plane direction ( $c$ axis) and the atomic displacements within the chain is in-phase-keeping the rigidity of $1 \mathrm{D}$ chains owing to no relative displacement among $\mathrm{TiS}_{3}$ atoms-we label this peak as I- $A_{\mathrm{g}}^{\text {rigid }}$. Overall, this mode can be pictured as two quasi-1D chains vibrating with respect to each other while keeping Ti-S bonding distance fixed within each chain. We note that this mode is absent in other 2D material systems such as transition metal dichalcogenides, TMDCs $\left(\mathrm{MoS}_{2}, \mathrm{WSe}_{2}\right.$ and so on), post-transition metal chalcogenides 
a

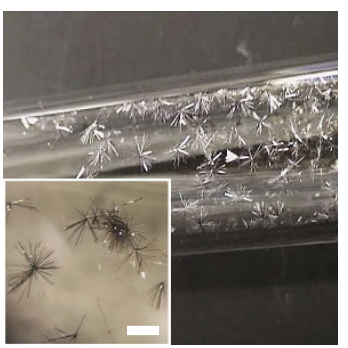

d

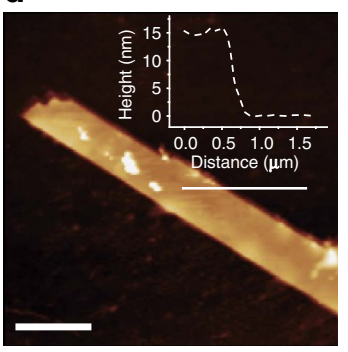

b

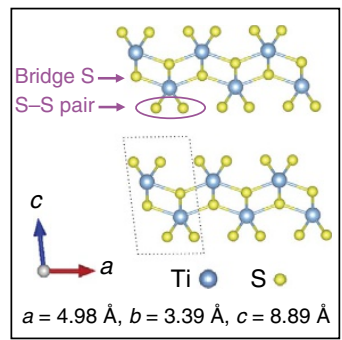

e

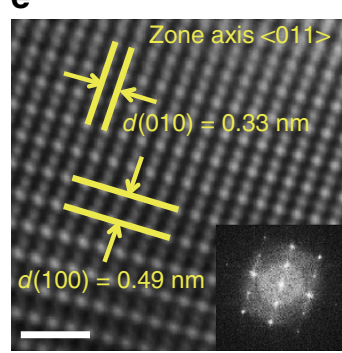

c

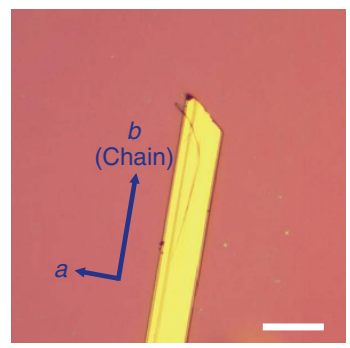

f

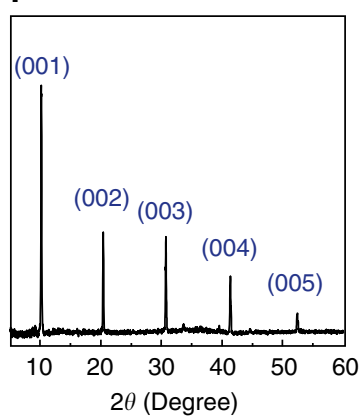

Figure 1 | Material synthesis and characterization. (a) Optical photograph of as-prepared $\mathrm{TiS}_{3}$ whiskers in the sealed quartz ampule and (inset) zoom-in images of $\mathrm{TiS}_{3}$ whiskers that were grown at the interior surface of quartz. Scale bar, $250 \mu \mathrm{m}$. (b) Schematic cross-section schematic view of TiS 3 chains along the $b$ axis, with $\mathrm{Ti}$ atoms in blue and $\mathrm{S}$ atoms in yellow. (c) Optical image of typical $\mathrm{TiS}_{3}$ whiskers exfoliated on $\mathrm{SiO}_{2} / \mathrm{Si}$ substrates with large geometrical anisotropy along the $b$ axis crystalline direction. Scale bar, $20 \mu \mathrm{m}$. (d) Atomic force microscope image of exfoliated TiS 3 whiskers with thickness $\sim 15 \mathrm{~nm}$. Scale bar, $1 \mu \mathrm{m}$. (e) High-resolution transmission electron microscopy image of $\mathrm{TiS}_{3}$ whiskers, and corresponding fast Fourier transform (FFT) image (inset). Scale bar, $1 \mathrm{~nm}$. (f) X-ray powder diffraction pattern of $\mathrm{TiS}_{3}$.

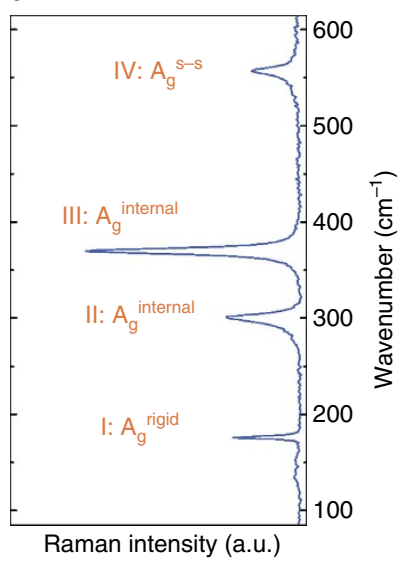

b

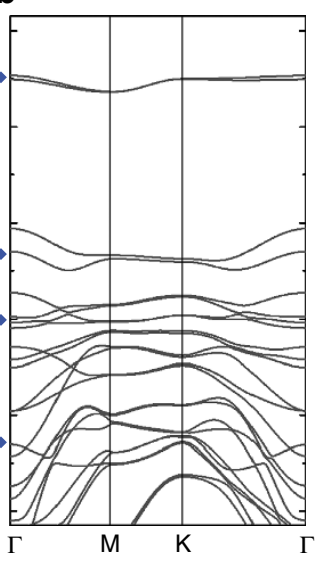

C

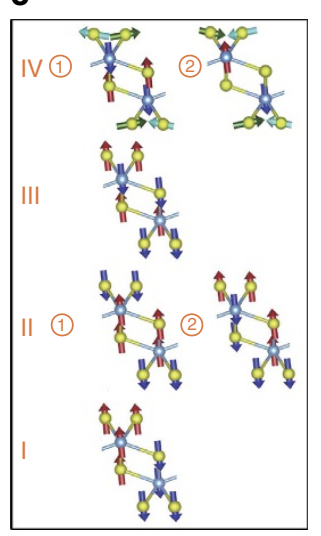

Figure 2 | Vibrational properties and phonon dispersion of $\mathbf{T i S}_{\mathbf{3}}$. (a) Raman spectrum of $\mathrm{TiS}_{3}$ whiskers measured in ambience at room temperature. (b) Phonon dispersion spectrum calculated using DFT of $\mathrm{TiS}_{3}$. The Raman active modes are highlighted by blue diamonds. (c) Corresponding Raman active optical modes in $\mathbf{a}$.

( $\mathrm{GaS}$ and GaSe), and even highly anisotropic TMDCs $\left(\mathrm{ReS}_{2}\right.$ and $\mathrm{ReSe}_{2}$ ) layers where $\mathrm{Re}$ atoms form Re-Re dimer chains.

In contrast to the rigid chain $\mathrm{I}-\mathrm{A}_{\mathrm{g}}^{\text {rigid }}$ mode, the peaks at $298 \mathrm{~cm}^{-1}$ (II-A $A_{\mathrm{g}}^{\text {internal}}$ ) and $370 \mathrm{~cm}^{-1}$ (III-A $\mathrm{A}_{\mathrm{g}}^{\text {internal}}$ ) involve vibration within each $\mathrm{TiS}_{3}$ layer, and these two vibrational modes are labelled as $A_{\mathrm{g}}^{\text {internal }}$ in relation to internal vibration in each layer. A closer look at $\Gamma \sim 300 \mathrm{~cm}^{-1}$ shows that two different optical branches coincide with each other. This implies that the II- $A_{\mathrm{g}}^{\text {internal }}$ mode consists of two degenerate modes as shown in Fig. $2 c$. Here the main difference between mode II- $A_{g}^{\text {internal }}$ - 1 (at

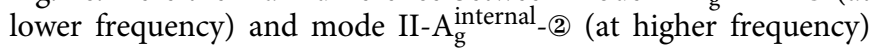

lies in the relative vibration direction between the $\mathrm{Ti}$ atoms, bridge $S$ atoms and $S-S$ pairs across the two prisms, as is shown in Fig. 2c. We note that the presence of two near degenerate Raman peaks results in much larger full-width half-maximum (FWHM) value $\left(12.1 \mathrm{~cm}^{-1}\right)$ compared with $\mathrm{I}_{-} \mathrm{A}_{\mathrm{g}}^{\text {rigid }}\left(3.3 \mathrm{~cm}^{-1}\right)$ and III-A $A_{\mathrm{g}}^{\text {internal }}\left(7.9 \mathrm{~cm}^{-1}\right)$. The large FWHM value of II- $A_{\mathrm{g}}^{\text {internal }}$ is attributed to degeneracy (presence of multiple) of modes at $\sim 298 \mathrm{~cm}^{-1}$. Similar to II-A $A_{\mathrm{g}}^{\text {internal }}$, the III-A $\mathrm{A}_{\mathrm{g}}^{\text {internal }}$ mode also involves vibration of atoms, making up the individual layers except that three $S$ atoms (both the bridge $S$ and $S-S$ pair) vibrate oppositely with the $\mathrm{Ti}$ atom in a single prism, whereas the other prism moves in a central symmetry way with it. 
In contrast to all the above modes, highest frequency peak at $556 \mathrm{~cm}^{-1}$ also appears doubly degenerate (Fig. 2b) with relatively large FWHM value $\left(12.3 \mathrm{~cm}^{-1}\right)$ as shown in Fig. 2a. Surprisingly, these peaks are predominantly made of in-plane out of phase motion of S-S pair and in part (minor) the out-of-plane motion of $\mathrm{Ti}$ and the bridge $\mathrm{S}$ atoms as shown in Fig. 2c. Considering the signature $\mathrm{S}-\mathrm{S}$ pair vibration, this peak is labelled as $I V-\mathrm{A}_{\mathrm{g}}^{\mathrm{S}-\mathrm{S}}$.

Pressure-dependent vibrational studies. To further understand the vibrational properties, we have performed pressuredependent Raman spectroscopy studies on $\mathrm{TiS}_{3}$ whiskers. Specifically, hydrostatic pressure was applied to the whiskers using a DAC, where the pressure media was a drop of methanolethanol (4:1) mixture and the pressure gauge was a small piece of ruby. Figure 3a displays the optical photograph of the DAC under the optical lens, and Fig. 3b shows the optical image of the $\mathrm{TiS}_{3}$ whiskers in the pressure media after loaded in the cell. Here the excitation (E) and detection (D) polarization was aligned parallel to the chain direction (E $\|\mathrm{D}\| b$ axis) to achieve high signal-to-noise ratio. Overall pressure trends, that is, negative $\mathrm{d} \omega / \mathrm{d} P$ values for IV-A $\mathrm{A}_{\mathrm{g}}^{\mathrm{S}-\mathrm{S}}$, lifting the degeneracy of Raman peaks and overall pressure dependence, were found to be independent from the polarization direction, but for completeness orthogonal polarization (E $\perp \mathrm{D} \| b$ axis) results are discussed and presented later in this section in Fig. 4c.

Figure $3 c$ shows the pressure-dependent Raman spectra of $\mathrm{TiS}_{3}$ from ambient pressure up to $26.3 \mathrm{GPa}$ in normal configuration (E $\|\mathrm{D}\| b$ axis). Rigid and internal modes, that is, I- $\mathrm{A}_{\mathrm{g}}^{\text {rigid }}$, II- $A_{\mathrm{g}}^{\text {internal }}$ and III- $A_{\mathrm{g}}^{\text {internal }}$, all stiffen with increasing pressure values because of increased interlayer interaction either stiffening the atomic bonds within each monolayers or increasing the interaction strength across 1D-like chain structures. The peak position of each individual mode scales linearly with pressure as depicted in Fig. 3e; thus, their frequency values can be described as $\omega(P)=\omega_{0}+(\mathrm{d} \omega / \mathrm{d} P) P$, where the slope value $(\mathrm{d} \omega / \mathrm{d} P)$ is given for each mode in Table 1 . It is also noteworthy that the $\mathrm{A}_{\mathrm{g}}^{\text {Rigid }}$ mode is significantly more sensitive to applied pressure $\left(\mathrm{d} \omega / \mathrm{d} P\left(\mathrm{~A}_{\mathrm{g}}^{\text {rigid }}\right)=3.82 \mathrm{~cm}^{-1} / \mathrm{GPa}>\mathrm{d} \omega / \mathrm{d} P\left(\mathrm{~A}_{\mathrm{g}}^{\text {internal }}\right) \approx 2 \mathrm{~cm}^{-1}\right.$

$\left.\mathrm{GPa}^{-1}\right)$ compared with internal modes likely because of easiness to increase interaction between moderately interacting $1 \mathrm{D}$-like chains-as opposed to strongly interacting atoms making up individual layers and chains. After the pressure is released, Raman spectrum of $\mathrm{TiS}_{3}$ returns back to its original (not pressurized) state, suggesting that pressure effects are reversible up to 26.3 $\mathrm{GPa}$, which is the highest pressure that can be attained in our set-up.

In contrast to other Raman peaks, the IV- $\mathrm{A}_{\mathrm{g}}^{\mathrm{S}-\mathrm{S}}$ mode softens with pressure, that is, $\mathrm{d} \omega / \mathrm{d} P\left(\mathrm{~A}_{\mathrm{g}}^{\mathrm{S}-\mathrm{S}}\right)<0$, which is rather unique compared with observations made on other layered systems. What is the origin of negative $\mathrm{d} \omega / \mathrm{d} P$ value of $\mathrm{A}_{\mathrm{g}}^{\mathrm{S}-\mathrm{S}}$ mode? Since IV- $\mathrm{A}_{\mathrm{g}}^{\mathrm{S}-\mathrm{S}}$ is the only mode with finite in-plane vibrational contribution from $\mathrm{S}-\mathrm{S}$ vibrations, it is possible that reduced interlayer distance at high pressures increases the S-S distance through an increase in S-Ti-S angle due to increase orbital interaction between adjacent layers. On the basis of this, we argue that the negative $\mathrm{d} \omega / \mathrm{d} P$ value is potentially related to softening of $\mathrm{S}-\mathrm{S}$ vibration, reducing the vibrational frequency of the $\mathrm{A}_{\mathrm{g}}^{\mathrm{S}-\mathrm{S}}$ mode.

To further understand the mode stiffness, we also performed quantitate analysis by calculating the Grüneisen parameters ${ }^{25}$ of a vibration mode, $\gamma$, which is defines as $\gamma=-(\partial \ln \omega /$

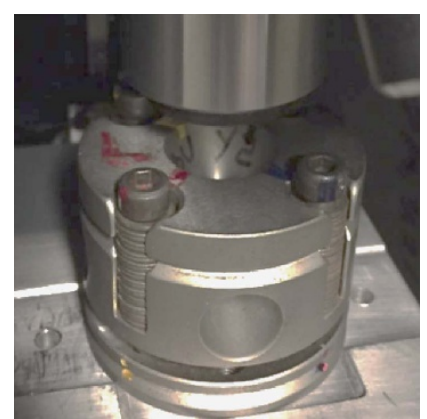

d

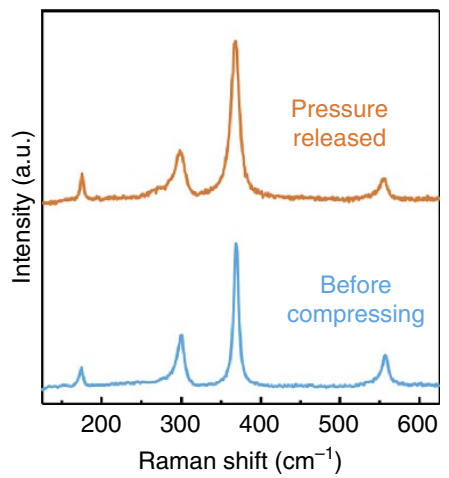

b

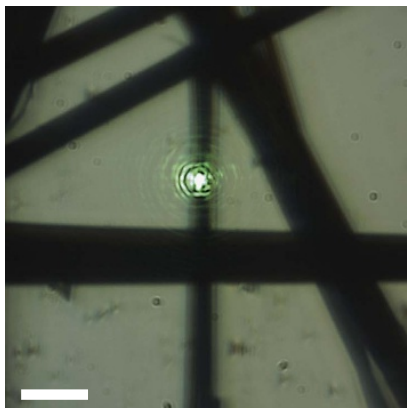

e

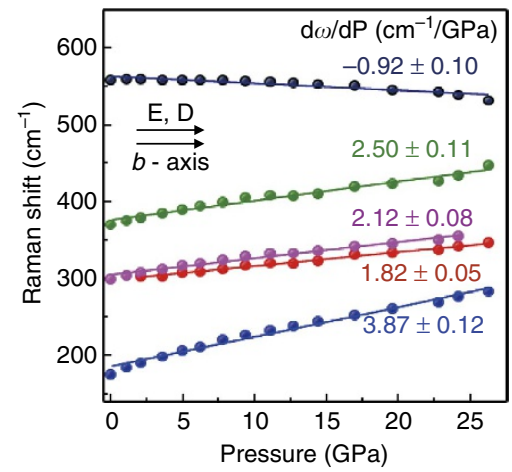

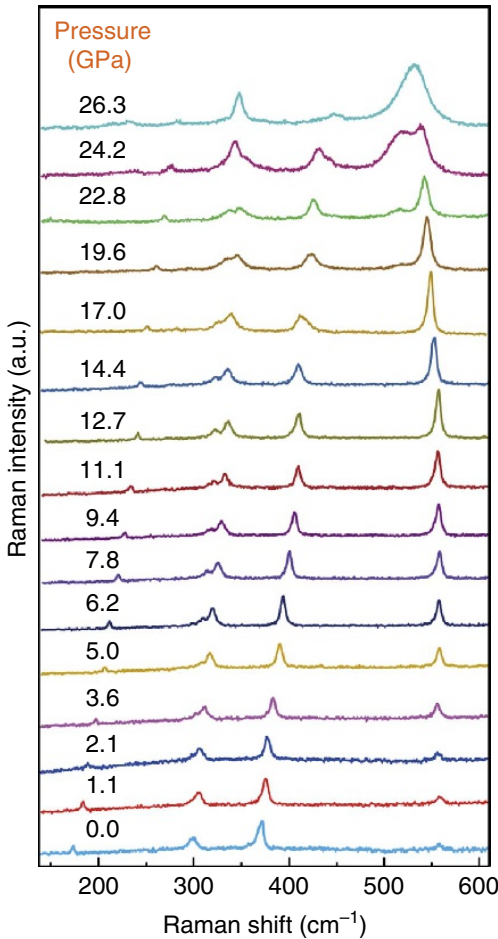

Figure 3 | The vibrational properties of TiS $_{\mathbf{3}}$ under hydrostatic pressure. (a) Optical photograph of the DAC under $\times 50$ optical lens in the experiment. (b) Optical image of $\mathrm{TiS}_{3}$ loaded inside the DAC. Scale bar, $1 \mathrm{~nm}$. (c) Raman spectra of $\mathrm{TiS}_{3}$ under increasing hydrostatic pressure up to $26.3 \mathrm{GPa}$; plots are offset for clarity. (d) A comparison between the Raman spectra of $\mathrm{TiS}_{3}$ before applying pressure and after releasing pressure from $26.3 \mathrm{GPa}$. (e) Raman peak position as a function of pressure. The excitation (E) and detection (D) polarization is in parallel to the $b$ axis. The dependence can be described as $\omega(P)=\omega_{0}+(\mathrm{d} \omega / \mathrm{d} P) P$. The $\omega_{0}$ and $\mathrm{d} \omega / \mathrm{d} P$ values are also shown in Table 1. 
a

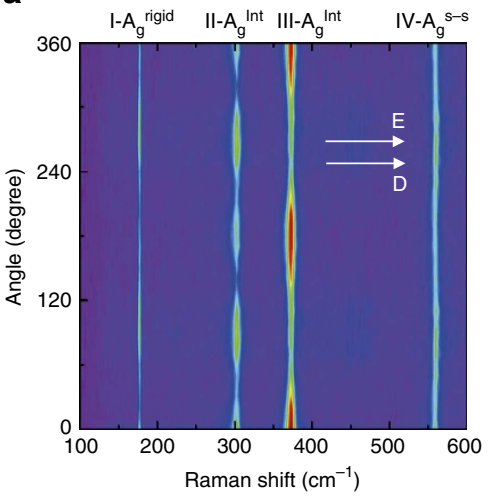

b

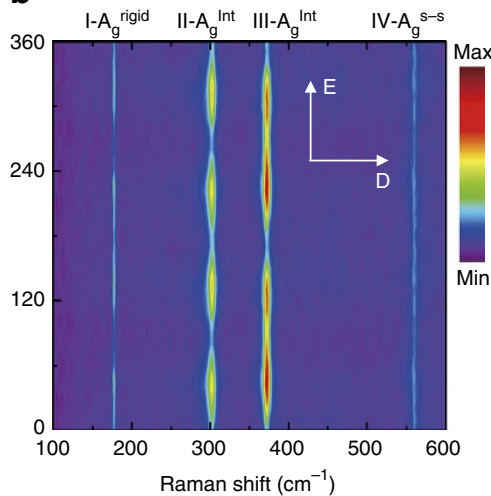

C

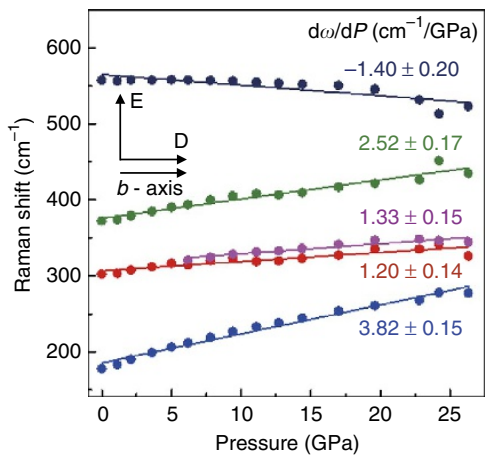

Figure 4 | Polarization dependence of Raman modes and their pressure dependence. Angle-resolved Raman spectroscopy (2D contour plots) for all the Raman peaks in the (a) normal $(E \| D)$ and $(\mathbf{b})$ orthogonal $(E \perp D)$ configuration. $E$ and $D$ represents the excitation direction and detection direction, respectively. (c) Evolution of Raman spectrum in orthogonal configuration displaying similar pressure-dependent trends and $\mathrm{d} \omega / \mathrm{d} P$ values.

Table 1 | Quantitative analysis of vibration properties of $\mathrm{TiS}_{3}$.

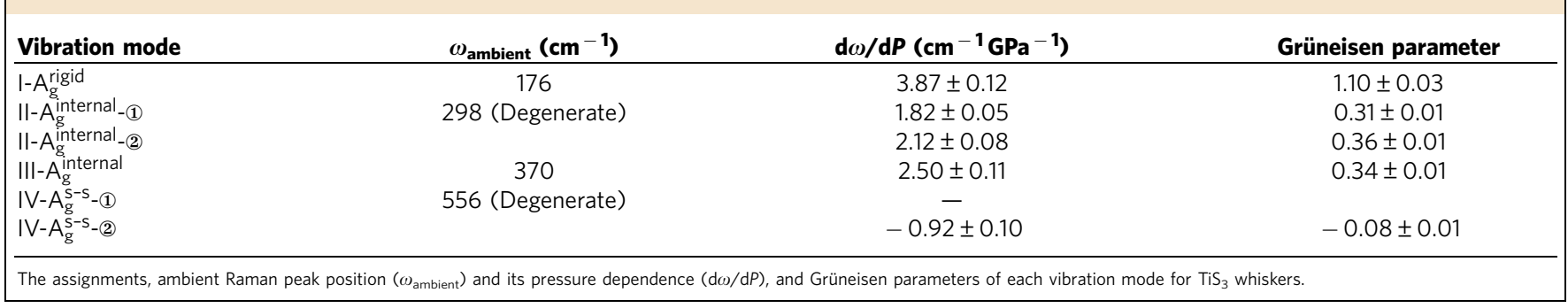

$\partial \ln V)=\left(\omega \chi_{T}\right)^{-1}(\partial \omega / \partial P)$, where $\omega$ is the frequency of the specific mode of interest, $V$ is the volume, $P$ is the pressure and $\chi_{T}=-V^{-1}(\partial V / \partial P)$ is the isothermal compressibility. In our case, the isothermal compressibility $\chi_{T}$ is replaced as $-c^{-1}(\partial c / \partial P)$ since our interested modes are along the $c$ axis. Thus, the final expression of the Grüneisen parameter is expressed as $\gamma=\left(-\omega c^{-1}(\partial c / \partial P)\right)^{-1}(\partial \omega / \partial P)$. The calculated Grüneisen parameters are also shown in Table 1 . We conclude that the I- $\mathrm{A}_{\mathrm{g}}^{\text {rigid }}$ mode is much stiffer than other modes because of its rigid chain nature.

Here we note that, related to particular Raman tensor of this material system, Raman modes show large angular variation with polarization angle. This is apparent from angle-resolved Raman spectroscopy data presented in $2 \mathrm{D}$ contour plots in Fig. 4a,b in the normal $(\mathrm{E} \| \mathrm{D})$ and orthogonal $(\mathrm{E} \perp \mathrm{D})$ configurations, respectively. The polarization angle is defined as the angle between the detection direction and $b$ axis. Depending on the polarization angle and polarization configuration, Raman intensity of different peaks goes through maxima and minima, and these two- or fourfold symmetry (two- or four-lobed) features are associated with the particular Raman tensors specific to this material system. These findings are in line with a recent study ${ }^{26}$ on $\mathrm{TiS}_{3}$, which has shown similar polarization effects on the III$\mathrm{A}_{\mathrm{g}}^{\text {Internal }}$ peak. In the same work, however, the other peaks demonstrated slightly less polarization-dependent response, in contrast to relatively strong polarization response measured in Fig. 4. This effect could possibly be attributed to presence of defects in the referenced work resulting in partial loss of crystalline anisotropy and much reduced angular response.

It is noteworthy to highlight that the polarization angle has no effect on the Raman peak position but has an impact on their relative intensity to each other as shown in Fig. 4a,b. Since internal mode (II- and III-A $\mathrm{A}_{\mathrm{g}}^{\text {Internal }}$ ) peaks are harder to identify at high pressures, normal polarization configuration was selected in Fig. $3 c, d$ to improve the signal from these modes (see Fig. $3 c$ at $0 \mathrm{GPa}$ and Fig. 4a). However, similar measurements in the orthogonal polarization direction also yield similar trends (that is, negative $\mathrm{d} \omega / \mathrm{d} P$ for IV- $\mathrm{A}_{\mathrm{g}}^{\mathrm{s}-\mathrm{s}}$ and peak splitting related to lifting degeneracy) and nearly matching $\mathrm{d} \omega / \mathrm{d} P$ values as shown in Fig. 4c. Similarly, the pressure dependence trends remain the same for thinner ( $\sim$ tens of nanometre thick) flakes as shown in Supplementary Fig. 1. However, we find it harder to collect sufficient and reliable signal at high pressures possibly because of the much weaker Raman intensity related to reduced material quantity as well as folding/wrinkling of ultrathin flakes in DAC liquid media causing unintentional flake folding.

Lifting the degeneracy of Raman modes. In addition to the unusual pressure dependence of the $\mathrm{A}_{\mathrm{g}}^{\mathrm{S}-\mathrm{S}}$ mode, we note that some of the peaks split into two components going from ambient to high pressures. In Fig. 5, we compare the shape of each Raman peak at 0 and $22.8 \mathrm{GPa}$. This figure convincingly shows that only II- $\mathrm{A}_{\mathrm{g}}^{\text {internal }}$ and IV-A $\mathrm{A}_{\mathrm{g}}^{\mathrm{S}-\mathrm{S}}$ modes split into two parts, whereas the other two retain their overall peak shape except minuscule broadening in their FWHM.

What is the origin of observed peak splitting for some of the peaks? This cannot be because of phase transition: peak splitting may (erroneously) be interpreted as 'pressure-induced phase transition'. However, careful pressure-dependent DFT calculations show that material retains its symmetry up to $30 \mathrm{GPa}$ (possibly above) and does not undergo phase transition. We, however, note that the pressure-induced splitting only occurs for doubly degenerate II- $\mathrm{A}_{\mathrm{g}}^{\text {internal }}$ and IV- $\mathrm{A}_{\mathrm{g}}^{\mathrm{S}-\mathrm{S}}$ modes (Fig. 2b). Since 
a

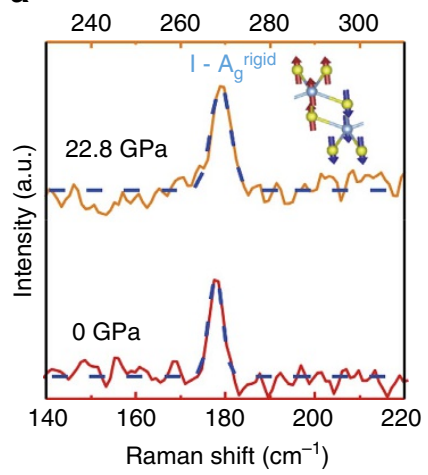

b

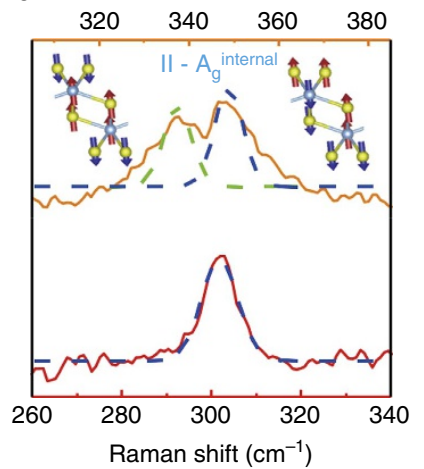

c

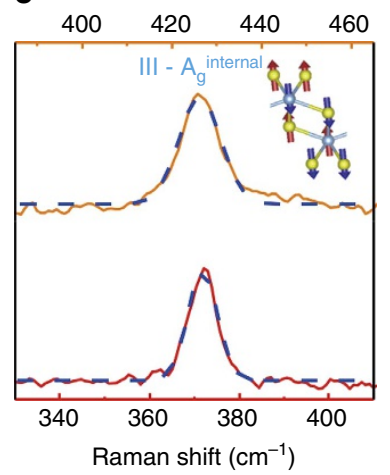

d

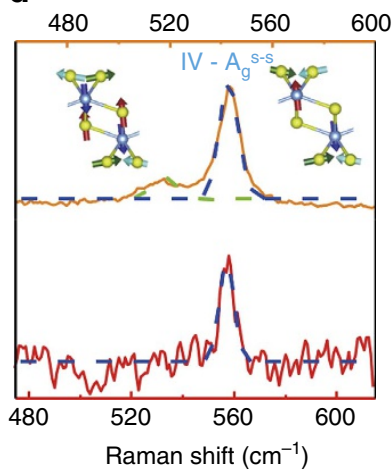

Figure 5 | A comparison between the shape of each Raman peak at $\mathbf{0}$ and $22.8 \mathrm{GPa}$. (a) I- $A_{g}^{\text {rigid }}$ mode, (b) II- $A_{g}^{\text {internal }}$ mode, (c) III- $A_{g}^{\text {internal }}$ mode (d) and IV-Ags mode.

each mode, including the degenerate ones, has different frequency dependence with applied pressure (different $\mathrm{d} \omega / \mathrm{d} P$ values), we argue that mode-specific splitting effect can be attributed to lifting the degeneracy of II- $\mathrm{A}_{\mathrm{g}}^{\text {internal }}$ and IV- $\mathrm{A}_{\mathrm{g}}^{\mathrm{S}-\mathrm{S}}$ modes by applied pressure. More explicitly, initially degenerate modes are in close proximity to each other within $\sim 5 \mathrm{~cm}^{-1}$, causing II- $A_{\mathrm{g}}^{\text {internal }}$ and IV-Ag $\mathrm{A}_{\mathrm{g}}^{\mathrm{S} S \mathrm{~S}}$ modes to have larger FWHM values compared with other modes; however, as the pressure is applied these modes start to separate from each other because of different $\mathrm{d} \omega / \mathrm{d} P$ values. Figure $5 \mathrm{~b}$ further shows the splitting of the II- $A_{\mathrm{g}}^{\text {internal }}$ mode; the FWHM values of the two peaks at $22.8 \mathrm{GPa}$, with the green dash line representing the II- $A_{g_{1}}^{\text {internal }}$ (1) mode and the blue dash line representing the II- $A_{g}^{\text {internal }}$ (2) mode, are comparable to that of the original degenerated II- $A_{\mathrm{g}}^{\text {internal }}$ mode. Similarly, blue and green dash lines in Fig. $5 \mathrm{~d}$ can be attributed to the degenerate modes forming the $\mathrm{A}_{\mathrm{g}}^{\mathrm{S}-\mathrm{S}}$ peak.

\section{Discussion}

To the best of our knowledge, results mark the first high-pressure DAC, extensive Raman and theoretical investigation on pseudo$1 \mathrm{D} \mathrm{TiS}_{3}$ material. Results are unexpected a priori as atomic motions of $\mathrm{TiS}_{3}$ vibrational modes are drastically different from other isotropic $\left(\mathrm{MoS}_{2}, \mathrm{WSe}_{2}\right.$, and so on) and even anisotropic materials $\left(\mathrm{ReS}_{2}, \mathrm{ReSe}_{2}\right.$ and black phosphorus). $\mathrm{TiS}_{3}$ exhibits various Raman active modes that are related to unique rigid chain vibrations (where chains vibrate as a whole), S-S molecular pair oscillations and conventional out-of-plane mode. Pressuredependent DAC Raman studies reveal that (i) the $\mathrm{A}_{\mathrm{g}}^{\mathrm{S}-\mathrm{S}}$ mode has a unique response to applied hydrostatic pressure with negative $\mathrm{d} \omega / \mathrm{d} P<0$ scaling and (ii) some modes appear to be degenerate, which is lifted only after pressure is exerted. Our theoretical studies by DFT and finite displacement methods further fundamental understanding of $\mathrm{TiS}_{3}$ and TMTC systems. Previously unknown vibrational properties of the material $\mathrm{TiS}_{3}$ may have broader impact impact towards understanding and interpreting thermal (thermal transport), structural (defects and impurities) and mechanical properties (strain) relevant for potential applications in optoelectronic and photonic devices.

\section{Methods}

Growth and characterization of $\mathrm{TiS}_{\mathbf{3}}$ whiskers. $\mathrm{TiS}_{3}$ whiskers were grown through the direct reaction of titanium wire and sulfur following the stoichiometric ratio. One gram of precursors was placed inside an evacuated and sealed quartz tube at a vacuum level of $\sim 10^{-5}$ Torr. The inner diameter for the tube was $15 \mathrm{~mm}$ and the length of it was $210 \mathrm{~mm}$. The loaded ampule was placed in a three-zone furnace and heated for 3 days. The end of ampule with source materials was heated up to $500^{\circ} \mathrm{C}$ while keeping a temperature gradient of $100^{\circ} \mathrm{C}$ at the other end. $\mathrm{TiS}_{3}$ whiskers were cleaved at the wall of the ampule at the cold end. The HR-TEM image was taken using JEOL JEM $2010 \mathrm{~F}$ at a voltage of $100 \mathrm{kV}$. The high crystallinity was further proved by the powder X-ray diffraction technique using $\mathrm{Cu} \mathrm{K} \alpha$ irradiation on Siemens D5000 X-Ray diffractometer. The Raman spectroscopy measurements of $\mathrm{TiS}_{3}$ whiskers were taken using a Renishaw InVia Raman microscope under $\times 50$ objective lens (numerical aperture $=0.95)$ using $488 \mathrm{~nm}$ laser as the excitation source, and the laser power was set to $0.8 \mathrm{~mW}$.

High-pressure Raman spectroscopy. The hydrostatic pressure was applied using a DAC. The $\mathrm{TiS}_{3}$ sample was placed in the pinhole of a gasket with $\sim 200 \mu \mathrm{m}$ diameter, where a drop of methanol-ethanol (4:1) mixture was used for pressure media. The pressure gauge was a small piece of ruby crystal deposited in the pinhole. Pressure was applied slowly to $26.3 \mathrm{GPa}$. The Raman spectra with increasing pressure were measured under long working distance $\times 50$ optical lens (numerical aperture $=0.42)$ using green laser $(\lambda=532 \mathrm{~nm}$ ) as the radiation source with a power of $1.3 \mathrm{~mW}$. The data were collected using an Acton 300i spectrograph and a back thinned Princeton Instruments liquid nitrogen-cooled charge-coupled device detector.

DFT calculations. The first-principles calculations were carried out in the framework of DFT as implemented in the Vienna $A b$ initio Simulation Package (VASP) ${ }^{27,28}$. Generalized gradient approximation (GGA) of PerdewBurke-Ernzerhof was employed for the exchange and correlation potentials ${ }^{29}$. A $10 \times 15 \times 5 \Gamma$-centred $\mathbf{k}$-point mesh was used for the Brillouin zone integration for the primitive unit cell of bulk $\mathrm{TiS}_{3}$. Frozen-core projector-augmented wave ${ }^{30,31}$ potentials were used with $500 \mathrm{eV}$ kinetic energy cutoff for the plane-wave expansion. The convergence criterion for energy is set to $10^{-5} \mathrm{eV}$ between two consecutive steps in the self-consistent field calculations. The atomic positions were relaxed until the Hellmann-Feynman forces are less than $10^{-4} \mathrm{eV} \AA^{-1}$. We used the DFT-D2 method of Grimme as implemented in VASP in order to describe the $\mathrm{vdW}$ interaction between $\mathrm{TiS}_{3}$ layers correctly ${ }^{32}$. The vibrational spectrum of the systems was investigated via ab initio phonon calculations. These were performed in the harmonic approximation by making use of the small displacement method in the harmonic approximation by
implemented in the PHON code

Data availability. The data that support the findings of this study are available from the corresponding author on request.

\section{References}

1. Wang, Q. H., Kalantar-Zadeh, K., Kis, A., Coleman, J. N. \& Strano, M. S. Electronics and optoelectronics of two-dimensional transition metal dichalcogenides. Nat. Nanotechnol. 7, 699-712 (2012).

2. Bhimanapati, G. R. et al. Recent advances in two-dimensional materials beyond graphene. ACS Nano 9, 11509-11539 (2015).

3. Akinwande, D., Petrone, N. \& Hone, J. Two-dimensional flexible nanoelectronics. Nat. Commun. 5, 5678 (2014).

4. Mak, K. F., Lee, C., Hone, J., Shan, J. \& Heinz, T. F. Atomically thin $\mathrm{MoS}_{2}$ : a new direct-gap semiconductor. Phys. Rev. Lett. 105, 136805 (2010).

5. Novoselov, K. S. et al. Electric field effect in atomically thin carbon films. Science 306, 666-669 (2004).

6. Xia, F., Wang, H. \& Jia, Y. Rediscovering black phosphorus as an anisotropic layered material for optoelectronics and electronics. Nat. Commun. 5, 4458 (2014).

7. Tran, V., Soklaski, R., Liang, Y. \& Yang, L. Layer-controlled band gap and anisotropic excitons in few-layer black phosphorus. Phys. Rev. B 89, 235319 (2014). 
8. Chenet, D. A. et al. In-plane anisotropy in mono- and few-layer $\mathrm{ReS}_{2}$ probed by Raman spectroscopy and scanning transmission electron microscopy. Nano Lett. 15, 5667-5672 (2015).

9. Aslan, O. B., Chenet, D. A., van der Zande, A. M., Hone, J. C. \& Heinz, T. F. Linearly polarized excitons in single- and few-layer $\mathrm{ReS}_{2}$ crystals. ACS Photon. 3, 96-101 (2016).

10. Liu, E. et al. Integrated digital inverters based on two-dimensional anisotropic $\mathrm{ReS}_{2}$ field-effect transistors. Nat. Commun. 6, 6991 (2015).

11. Dai, J., Li, M. \& Zeng, X. C. Group IVB transition metal trichalcogenides: a new class of 2D layered materials beyond graphene. Wiley Interdiscip. Rev. 6, 211-222 (2016).

12. Galliardt, D. W., Nieveen, W. R. \& Kirby, R. D. Lattice properties of the linear chain compound $\mathrm{TiS}_{3}$. Solid State Commun. 34, 37-39 (1980).

13. Furuseth, S., Brattas, L. \& Kjekshus, A. On the crystal structures of $\mathrm{TiS}_{3}, \mathrm{ZrS}_{3}, \mathrm{ZrSe}_{3}, \mathrm{ZrTe}_{3}, \mathrm{HfS}_{3}$, and $\mathrm{HfSe}_{3}$. Acta Chem. Scand. 29, 623-631 (1975).

14. Gorlova, I. G., Pokrovskii, V. Y., Zybtsev, S. G., Titov, A. N. \& Timofeev, V. N. Features of the conductivity of the quasi-one-dimensional compound $\mathrm{TiS}_{3}$. J. Exp. Theor. Phys. 111, 298-303 (2010).

15. Ferrer, I. J., Maciá, M. D., Carcelén, V., Ares, J. R. \& Sánchez, C. On the photoelectrochemical properties of $\mathrm{TiS}_{3}$ films. Energy Proc. 22, 48-52 (2012).

16. Ferrer, I. J., Ares, J. R., Clamagirand, J. M., Barawi, M. \& Sánchez, C. Optical properties of titanium trisulphide $\left(\mathrm{TiS}_{3}\right)$ thin films. Thin Solid Films 535, 398-401 (2013).

17. Dai, J. \& Zeng, X. C. Titanium trisulfide monolayer: theoretical prediction of a new direct-gap semiconductor with high and anisotropic carrier mobility. Angew. Chem. Int. Ed. 54, 7572-7576 (2015).

18. Molina-Mendoza, A. J. et al. Electronic bandgap and exciton binding energy of layered semiconductor $\mathrm{TiS}_{3}$. Adv. Electron. Mater. 1, 1500126 (2015).

19. Island, J. O. et al. Ultrahigh photoresponse of few-layer $\mathrm{TiS}_{3}$ nanoribbon transistors. Adv. Opt. Mater. 2, 641-645 (2014).

20. Lipatov, A. et al. Few-layered titanium trisulfide $\left(\mathrm{TiS}_{3}\right)$ field-effect transistors. Nanoscale 7, 12291-12296 (2015).

21. Island, J. O. et al. $\mathrm{TiS}_{3}$ transistors with tailored morphology and electrical properties. Adv. Mater. 27, 2595-2601 (2015).

22. Gard, P., Cruege, F., Sourisseau, C. \& Gorochov, O. Single-crystal micro-Raman studies of $\mathrm{ZrS3}, \mathrm{TiS}_{3}$ and several $\mathrm{Zr}_{1-\mathrm{x}} \mathrm{TixS}_{3}$ compounds $(0<\mathrm{x} \leqslant 0.33)$. J. Raman Spectrosc. 17, 283-288 (1986).

23. Wieting, T. J., Grisel, A. \& Levy, F. Lattice dynamical study of the lowdimensional type B compounds $\mathrm{TiS}_{3}, \mathrm{HfSe}_{3}$ and $\mathrm{ZrTe}_{3}$. Phys. B $+\mathrm{C} \mathrm{105}$, 366-369 (1981).

24. Brattas, L. \& Kjekshus, A. Properties of compounds with $\mathrm{ZrSe}_{3}$ type structure. Acta Chem. Scand. 26, 3441-3449 (1972).

25. O'Neal, K. R. et al. High pressure vibrational properties of $\mathrm{WS}_{2}$ nanotubes. Nano Lett. 16, 993-999 (2016).

26. Island, J. O. et al. Titanium trisulfide $\left(\mathrm{TiS}_{3}\right)$ : a $2 \mathrm{D}$ semiconductor with quasi-1D optical and electronic properties. Sci. Rep. 6, 22214 (2016).

27. Kresse, G. \& Furthmüller, J. Efficiency of ab-initio total energy calculations for metals and semiconductors using a plane-wave basis set. Comput. Mater. Sci. 6, 15-50 (1996).

28. Kresse, G. \& Furthmüller, J. Efficient iterative schemes for ab initio total-energy calculations using a plane-wave basis set. Phys. Rev. B 54, 11169-11186 (1996).
29. Perdew, J. P., Burke, K. \& Ernzerhof, M. Generalized gradient approximation made simple. Phys. Rev. Lett. 77, 3865-3868 (1996).

30. Blöchl, P. E. Projector augmented-wave method. Phys. Rev. B 50, 17953-17979 (1994).

31. Kresse, G. \& Joubert, D. From ultrasoft pseudopotentials to the projector augmented-wave method. Phys. Rev. B 59, 1758-1775 (1999).

32. Grimme, S. Semiempirical GGA-type density functional constructed with a long-range dispersion correction. J. Comput. Chem. 27, 1787-1799 (2006).

33. Alfe, D. PHON: A program to calculate phonons using the small displacement method. Comput. Phys. Commun. 180, 2622-2633 (2009).

\section{Acknowledgements}

S.T. acknowledges support from the National Science Foundation (DMR-1552220) and (CMMI-1561839). F.M.P., H.S. and E.T. were supported by the Flemish Science Foundation (FWO-Vl). Computational resources were partially provided by TUBITAK ULAKBIM, High Performance and Grid Computing Center (TR-Grid e Infrastructure). H.S. acknowledges support from Bilim Akademisi-The Science Academy, Turkey under the BAGEP programme. F.P. acknowledges the funding from Flemish Science Foundation (FWO-Vl). K.W. acknowledges helpful discussions with H. Cai, W. Kong and X. Meng. We gratefully acknowledge the use of facilities within the LeRoy Eyring Center for Solid State Science at Arizona State University.

\section{Author contributions}

K.W. and E.S. performed high-pressure Raman spectroscopy, the X-ray powder diffraction measurements and wrote the manuscript. A.P. and B.C. preformed angle-dependent Raman measurements. B.C. performed AFM measurements. X.F. and D.P.W. grew the $\mathrm{TiS}_{3}$ whiskers. E.T., H.S. and F.P. have performed theoretical calculations. B.C., K.W. and T.A. have performed TEM measurements. S.T. designed experiments and wrote the manuscript.

\section{Additional information}

Supplementary Information accompanies this paper at http://www.nature.com/ naturecommunications

Competing financial interests: The authors declare no competing financial interests.

Reprints and permission information is available online at http://npg.nature.com/ reprintsandpermissions/

How to cite this article: $\mathrm{Wu}, \mathrm{K}$. et al. Unusual lattice vibration characteristics in whiskers of the pseudo-one-dimensional titanium trisulfide $\mathrm{TiS}_{3}$. Nat. Commun. 7, 12952 doi: $10.1038 /$ ncomms12952 (2016)

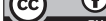

This work is licensed under a Creative Commons Attribution 4.0 International License. The images or other third party material in this article are included in the article's Creative Commons license, unless indicated otherwise in the credit line; if the material is not included under the Creative Commons license, users will need to obtain permission from the license holder to reproduce the material. To view a copy of this license, visit http://creativecommons.org/licenses/by/4.0/

(C) The Author(s) 2016 